\title{
Caracterização morfológica da região de transição entre o intestino delgado e o grosso no javali (Sus scrofa, L., 1758)
}

\author{
Ana Paula VIDOTTI ${ }^{1}$ \\ Liberato John Alphonse DI \\ $\mathrm{DIO}^{2}$ \\ Isaura Maria Mesquita \\ PRADO $^{3}$
}

\section{(1)Correspondência para:}

Rua Professor Guido Inácio Bersch, nº 60 ap. 04-Jd. Universitário-Maringá, Paraná, Brasil-CEP 87020-250 -

e-mail:avidotti@usp.br

(2) In memorian

Recebido para publicação: 10/03/2004 Aprovado para publicação: 27/03/2007

\author{
1 - Departamento de Cirurgia, Faculdade de Medicina Veterinária e \\ Zootecnia, Universidade de São Paulo, São Paulo-SP \\ 2 - Departamento de Cirurgia, Escola de Medicina, Universidade de Santo \\ Amaro, São Paulo - SP \\ 3 - Departamento de Ciências Morfofisiológicas, Universidade Estadual de \\ Maringá, Maringá - PR
}

\section{Resumo}

Estudou-se a morfologia da região de transição entre os intestinos delgado e grosso de 32 javalis (Sus scrofa, L., 1758), geneticamente selecionados, de ambos os sexos, entre jovens e adultos. O exame macroscópico contemplou - conformação e direção da saliência do íleo no intestino grosso, do óstio ileal, do íleo terminal e dos intestinos ceco e cólon ascendente, bem como as medidas do íleo no intestino grosso. Os resultados foram submetidos ao teste de Wilcoxon $(\alpha \leq 0,05)$. A terminação do íleo no intestino grosso constituía uma junção íleo-ceco-cólica. O ceco estava constituído por 3 (três) tênias: uma lateral e uma medial, contínuas, e uma ventral, esta terminando antes do ápice deste segmento. A superfície externa do cólon ascendente apresentava duas tênias: lateral e medial, em continuidade com as tênias homônimas do ceco. O íleo terminal dos javalis apresentou morfologia cilindróide em 100\% dos casos. Em todos os casos observamos a eminência ileal em forma papilar - papila ileal (papilla ilealis) - voltada para o intestino grosso, com direção oblíqua. No ápice da papila observamos um óstio central (ostium ileale), relativamente fechado e de forma estrelada ( $75 \%$ dos casos), ou relativamente aberto e de forma ovalada (25\%). Nossos achados demonstraram diferentes morfologias em relação a outros animais, constituindo mais uma indicação contra a generalização desta região nos mamíferos.
Palavras-chave:

Morfologia. Intestinos. Animais silvestres. Anatomia. Javali. Sus scrofa (L., 1758).

\section{Introdução}

A região de transição entre os intestinos delgado e grosso varia marcadamente entre as diferentes espécies ${ }^{1}$ e muitos estudos têm sido feitos no sentido de conhecer suas características morfológicas e fisiológicas em animais domésticos e de laboratório. Contudo, poucos estudos têm sido desenvolvidos em animais silvestres.

Presumivelmente, a morfologia desta região está relacionada com o tipo de digestão do animal, de modo que naqueles animais que apresentam digestão total ou parcial no ceco, a terminação do íleo ocorre neste órgão ${ }^{1}$.

A porção terminal do íleo é de grande importância para o processo digestivo ${ }^{2,3,4}$, sendo consenso que sua morfologia está, direta ou indiretamente, relacionada com a função dos intestinos delgado e grosso ${ }^{5,6,7}$. O íleo dos animais domésticos é limitado pela prega íleo-cecal, contudo, apesar da importância do íleo terminal, a delimitação deste segmento não está bem definida na literatura. Em suínos alguns autores admitem como sendo os dez centímetros distais do íleo ${ }^{8,9,10}$. As análises macro e mesoscópicas do íleo mostraram diferentes formas para o íleo terminal de suínos - cilíndrica, 
infundibular ou ampular, o que estaria relacionado com o momento funcional do órgão ${ }^{9,10}$.

Estudando a morfologia do estômago e do intestino de cinco javalis, Schäppi ${ }^{11}$ descreveu que o íleo termina no intestino grosso ao nível da transição cecocólica. O íleo foi caracterizado, basicamente, pela diminuição do calibre e pela maior espessura de sua túnica muscular em comparação com os demais segmentos do intestino delgado ${ }^{(11)}$. Contudo este autor não faz referência quanto à caracterização do íleo terminal.

O javali é uma espécie produtora de carne e tanto seu consumo, quanto sua produção vem crescendo a cada dia. Muitos produtores optam pela criação de espécimes mestiços ("javaporco"), com o objetivo principal de facilitar o manejo, e progressos têm ocorrido nos estudos genéticos sobre esta espécie. Entretanto, pouco se conhece sobre o sistema digestório destes animais, objeto de maior importância não só para o desenvolvimento e adequação da alimentação como, também, para estudos fisiológicos, parasitológicos e patológicos.

Neste trabalho pretende-se estudar a limitação morfológica da junção entre os intestinos delgado e grosso de javalis, geneticamente puros, a morfologia da saliência ileal e dos segmentos do íleo terminal e do intestino grosso vizinhos.

\section{Material e Método}

Foram utilizados 32 javalis (Sus scrofa, L., 1758), geneticamente selecionados (36 cromossomos), de ambos os sexos, incluindo jovens e adultos, provenientes de criadouros do interior do Estado de São Paulo (Fazenda São Pedro - registro IBAMA 2/35/96/0314-5, e Estância Ires - registro IBAMA 2/35/1997000401-8). As amostras, compreendendo o íleo, o intestino ceco e 15-20 centímetros iniciais do cólon ascendente, foram obtidas aproximadamente 10 minutos após a morte do animal.

Após lavagem cuidadosa, as peças tiverem o ceco aberto longitudinalmente, entre as tênias lateral e medial, iniciando a incisão no cólon ascendente, percorrendo todo o ceco até a união das tênias laterais, de modo a expor a terminação do íleo no intestino grosso. A descrição morfológica compreendeu características macroscópicas gerais - conformação e direção da saliência do íleo no intestino grosso, do óstio ileal, do íleo terminal e dos segmentos adjacentes dos intestinos ceco e cólon ascendente. A seguir, foram tomadas algumas medidas, incluindo a altura dos contornos cranial e caudal da saliência e diâmetro do ápice. Os resultados foram submetidos ao teste de Wilcoxon ${ }^{12}$ para dados pareados, com aproximação à curva normal, a fim de comparar as medidas entre as peças frescas e fixadas. Estabeleceu-se em 0,05 ou $5 \%$ $(\alpha \leq 0,05)$ o nível de rejeição da hipótese de nulidade. Realizadas as observações, o material foi conservado em solução de formol a $10 \%$ e as amostras mais representativas foram fotografadas.

\section{Resultados}

O íleo estava fixado ao ceco pela prega íleo-cecal, a qual se inseria por toda a extensão da tênia ventral do ceco. A porção terminal do íleo desembocava no intestino grosso, ao nível da junção ceco-cólica, formando um ângulo agudo com o ceco. $O$ íleo invaginava-se na luz do intestino grosso, levando consigo as paredes do cólon ascendente e do ceco, que formavam, respectivamente, os contornos cranial (ileocólico) e caudal (íleo-cecal) da saliência ileal (Figuras 1 e 2). A porção terminal do íleo tinha forma de cilindro em $100 \%$ dos casos - "íleo terminal cilindróide" " 5 . O ceco estava constituído por três tênias: uma lateral e uma medial, contínuas, e uma ventral terminando antes do ápice deste segmento. A superfície externa do cólon ascendente apresentava duas tênias: lateral e medial, em continuidade com as tênias homônimas do ceco. Exatamente na transição ceco-cólica, observou-se a saliência do íleo no intestino grosso, que se apresentou, em todos os casos, de forma papilar - papila ileal (papilla ilealis) ${ }^{13}$ 
(Figuras 1 e 2) - voltada para o intestino grosso, com direção oblíqua. As dimensões da papila ileal são apresentadas na tabela 1. As correlações feitas para as medidas da papila ileal entre peças frescas e fixadas são apresentadas no quadro 1. Na maioria dos casos $(75 \%)$, a papila apontava para o lume cecal; em 18,75\% dispunha-se centralmente e, no restante dos casos (6,25\%), a papila ileal apontava, suavemente, para o lume do cólon ascendente. No ápice da papila observamos um óstio central (ostium ileale) $)^{(13)}$, relativamente fechado e de forma estrelada (75\% dos casos) (Figura 1), ou relativamente aberto e de forma ovalada (25\%) (Figura 2). Observou-se, nitidamente, em 31,25\% dos casos a presença de uma borda proeminente da papila a partir do seu óstio (Figura 1).

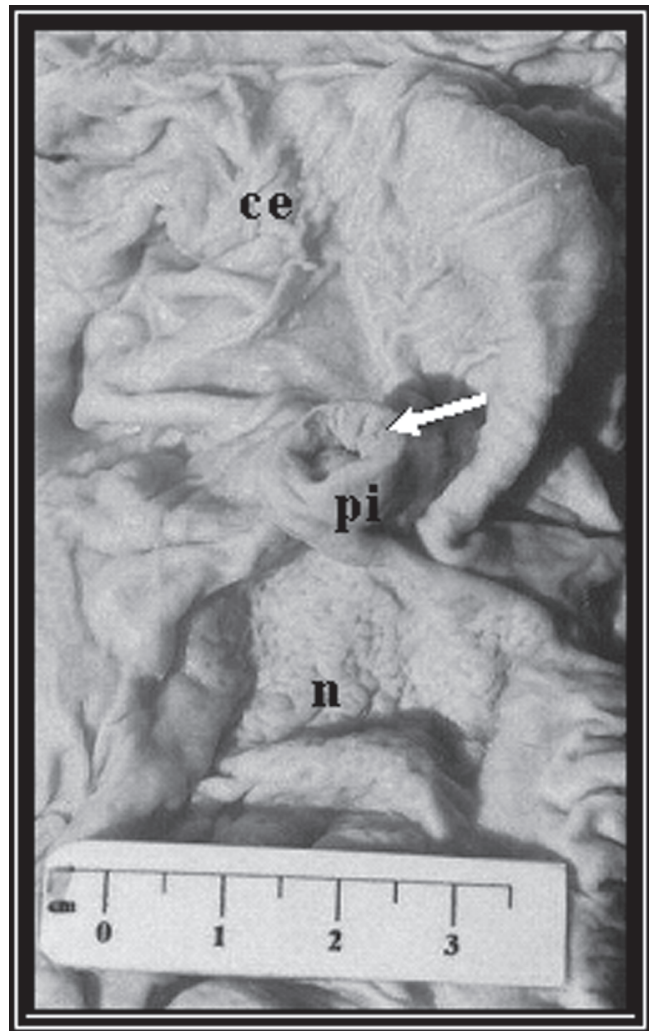

Figura 1 - Vista interna da junção íleo-ceco-cólica de javali (Sus scrofa, L., 1758), macho, evidenciando a papila ileal (pi), com óstio ovalado. Observe a borda proeminente da papila ileal a partir do seu óstio (seta). Note pregas circulares da túnica mucosa do ceco (ce) e nódulos linfáticos agregados na túnica mucosa do cólon ascendente (n) (escala em centímetros)

\section{Discussão}

O íleo de javalis (Sus scrofa, L., 1758) desembocava no intestino grosso, formando um ângulo agudo com o ceco, em $100 \%$ dos casos, confirmando o observado para a mesma espécie por Schäppi ${ }^{11}$. Esta angulação foi também indicada para a espécie suína ${ }^{9,10,14,15,16,17}$, e para a espécie humana ${ }^{5,18,19}$.

Devido à importância funcional dos últimos centímetros do íleo para humanos ${ }^{5,20,21}$, e para suínos ${ }^{8,9,10}$, procuramos neste trabalho, delimitar morfologicamente o "íleo terminal" do javali. Embora tenha sido indicado que o comprimento total do íleo do javali varia de 18 a $29 \mathrm{~cm}^{11}$, não encontramos nenhum registro sobre o íleo terminal. A julgar pelo seu descendente suíno e por seu hábito alimentar onívoro,

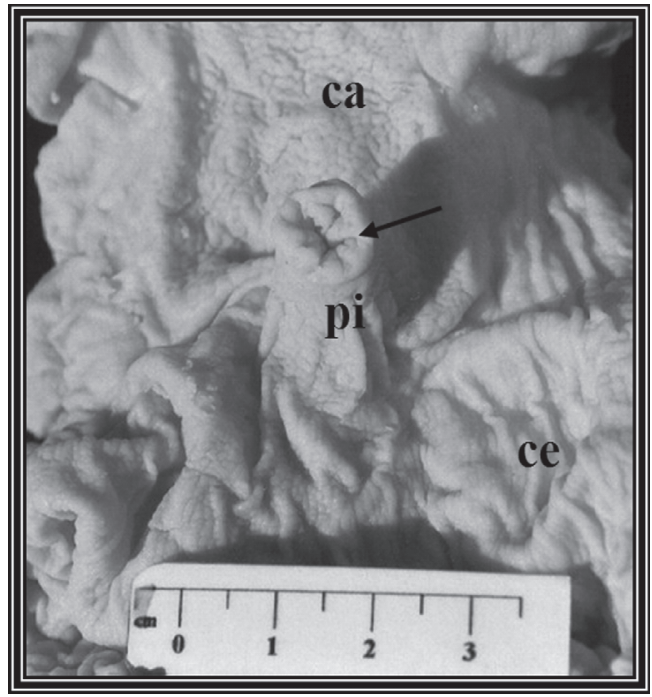

Figura 2 - Vista interna da junção íleo-ceco-cólica de javali (Sus scrofa, L., 1758), fêmea, mostrando o contorno caudal da papila ileal, esta com óstio estrelado (seta) relativamente fechado. Note pregas da túnica mucosa, longitudinalmente dispostas, neste contorno. Cólon ascendente (ca); ceco (ce); papila ileal (pi) (escala em centímetros)

admitimos neste trabalho o íleo terminal de javalis como os últimos $10 \mathrm{~cm}$ distais do íleo, como indicado para suínos ${ }^{8,9,10}$ e para a espécie humana ${ }^{5}$. A porção terminal do íleo de javalis tinha forma de cilindro - íleo terminal cilindróide ${ }^{5}$. No homem o íleo terminal apresentava variação na sua conformação 20,21 "ampola de Busi";22 
Tabela 1 - Javalis (Sus scrofa, L., 1758) segundo as dimensões (em centímetros) da altura dos contornos cranial e caudal e do diâmetro doápice da papila ileal nas peças frescas e fixadas - São Paulo - 2003

\begin{tabular}{|c|c|c|c|c|c|c|}
\hline \multirow{2}{*}{$\begin{array}{c}\text { ANIMAI } \\
\mathbf{N}^{\mathbf{0}}\end{array}$} & \multicolumn{2}{|c|}{$\begin{array}{l}\text { ALTURA CONTORNO } \\
\text { CRANIAL }\end{array}$} & \multicolumn{2}{|c|}{$\begin{array}{c}\text { ALTURA CONTORNO } \\
\text { CAUDAL }\end{array}$} & \multicolumn{2}{|c|}{$\begin{array}{l}\text { DIÂMETRO } \\
\text { DO ÁPICE }\end{array}$} \\
\hline & $\overline{\text { Peça Fresca }}$ & Peça Fixada & Peça Fresca & Peça Fixada & Peça Fresca & Peça Fixada \\
\hline 1 & 2,00 & 2,05 & 2,85 & 2,61 & 2,04 & 1,90 \\
\hline 2 & 2,12 & 2,22 & 2,43 & 2,51 & 1,75 & 1,68 \\
\hline 3 & 2,15 & 1,92 & 2,17 & 1,98 & 2,23 & 1,72 \\
\hline 4 & 1,74 & 1,86 & 1,95 & 1,70 & 2,09 & 1,70 \\
\hline 5 & 2,06 & 2,10 & 1,14 & 1,40 & 1,79 & 1,57 \\
\hline 6 & 1,56 & 1,60 & 1,92 & 1,16 & 2,16 & 1,23 \\
\hline 7 & 1,44 & 1,46 & 1,53 & 1,50 & 2,00 & 1,40 \\
\hline 8 & 1,54 & 1,61 & 1,45 & 1,46 & 1,85 & 1,40 \\
\hline 9 & 1,63 & 1,75 & 1,56 & 1,10 & 1,69 & 1,67 \\
\hline 10 & 0,98 & 1,06 & 1,04 & 1,11 & 1,75 & 1,28 \\
\hline 11 & 1,38 & 1,60 & 1,55 & 1,40 & 1,33 & 1,18 \\
\hline 12 & 0,94 & 1,02 & 1,07 & 1,22 & 2,05 & 1,06 \\
\hline 13 & 1,02 & 1,50 & 1,20 & 1,00 & 1,81 & 1,00 \\
\hline 14 & 2,30 & 2,70 & 1,19 & 1,02 & 2,12 & 1,20 \\
\hline 15 & 2,18 & 2,26 & 1,39 & 1,50 & 1,92 & 0,96 \\
\hline 16 & 1,80 & 2,12 & 1,18 & 1,20 & 1,96 & 1,11 \\
\hline 17 & 1,74 & 1,80 & 1,20 & 1,20 & 2,18 & 1,20 \\
\hline 18 & 1,42 & 2,00 & 1,10 & 1,21 & 1,94 & 1,07 \\
\hline 19 & 1,97 & 2,01 & 0,98 & 1,30 & 2,03 & 1,26 \\
\hline 20 & 2,21 & 2,50 & 1,10 & 1,70 & 2,12 & 1,22 \\
\hline 21 & 1,77 & 2,26 & 0,76 & 1,34 & 1,87 & 1,03 \\
\hline 22 & 1,14 & 1,98 & 2,00 & 1,61 & 2,20 & 1,24 \\
\hline 23 & 1,50 & 2,05 & 1,00 & 1,20 & 1,02 & 1,40 \\
\hline 24 & 2,30 & 2,10 & 1,18 & 1,00 & 1,05 & 1,40 \\
\hline 25 & 2,00 & 2,20 & 1,56 & 1,36 & 1,00 & 1,20 \\
\hline 26 & 2,20 & 2,70 & 1,50 & 1,25 & 1,00 & 1,16 \\
\hline 27 & 1,83 & 2,00 & 1,20 & 1,04 & 1,00 & 1,18 \\
\hline 28 & 1,70 & 2,03 & 0,90 & 1,40 & 0,94 & 1,20 \\
\hline 29 & 1,31 & 1,42 & 1,58 & 1,60 & 1,02 & 1,34 \\
\hline 30 & 2,40 & 2,68 & 2,00 & 2,64 & 0,90 & 1,20 \\
\hline 31 & 1,40 & 2,00 & 1,10 & 1,60 & 1,20 & 1,60 \\
\hline 32 & 2,05 & 2,84 & 1,50 & 1,90 & 1,20 & 1,31 \\
\hline Média & 1,74 & 1,98 & 1,45 & 1,48 & 1,66 & 1,31 \\
\hline
\end{tabular}

“órgão antro-ileal"; 5,7,19,23,24,25,26,27, tendo sido caracterizada três conformações - íleo cilindróide, ampular, infundibular ${ }^{5}$. Estas três formas foram, também, indicadas para suínos ${ }^{9,10}$. Acredita-se que a variedade na forma do íleo possa estar relacionada com a atividade muscular intestinal no momento que precede a morte do animal ${ }^{9,10}$. O confronto de nossos resultados com as observações dos demais autores é um indicativo da necessidade de estudos morfológicos e funcionais sobre a inervação intrínseca e extrínseca, bem como estudos endócrinos desta região.

O ceco, em todos os casos, apresentava três tênias - tênias lateral e medial, mais definidas e contínuas, e tênia ventral que terminava antes do ápice do ceco a mais ou menos $2 \mathrm{~cm}$ da confluência das demais. Nossos resultados divergiram dos 
Fresca X Fixada - z crítico $=1,64$

\begin{tabular}{|l|c|c|c|}
\hline VARIÁVEIS & $\mathbf{T}^{(1)}$ & $\boldsymbol{Z}^{(2)}$ & $\mathbf{p}^{(3)}$ \\
\hline $\mathbf{A C C F}^{(4)} \& \mathbf{A C C X}^{(5)}$ & 35,5 & 4,272708 & ${ }^{*(10)} 0,0000194$ \\
\hline $\mathbf{A C D F}^{(6)} \& \mathbf{A C D X}^{(7)}$ & 231 & 0,333141 & 0,73903 \\
\hline $\mathbf{D F}^{(8)} \& \mathbf{D X}^{(9)}$ & 100 & 3,066626 & ${ }^{*(11)} 0,002167$ \\
\hline
\end{tabular}

${ }^{(1)}$ menor soma de postos do mesmo sinal. ${ }^{(2)}$ valores críticos de T. ${ }^{(3)}$ probabilidade associada a ocorrência, sob $\mathrm{H}_{0}$, de um valor tão extremo quanto ou mais extremo do que o valor observado. ${ }^{(4)}$ atura do contorno cranial nas peças frescas. ${ }^{(5)}$ altura do contorno cranial nas peças fixadas. ${ }^{(6)}$ altura do contorno caudal nas peças frescas. ${ }^{\left({ }^{(7)}\right.}$ altura do contorno caudal nas peças fixadas. ${ }^{(8)}$ diâmetro do ápice da papila nas peças frescas. ${ }^{(9)}$ diâmetro do ápice da papila nas peças fixadas. ${ }^{*}{ }^{* 10)} \mathrm{e}^{*(11)}$ valores significantes ao nível de $\alpha \leq 0,05$.

Quadro 1 - Teste de Wilcoxon para dados pareados comparando as dimensões da papila ileal entre as peças frescas eas fixadas dos javalis (Sus scrofa, L., 1758) - São Paulo - 2003

descritos para a mesma espécie ${ }^{11}$ e para a espécie suína ${ }^{9,17}$, pois as três tênias alcançavam o ápice do órgão. A superfície externa do cólon ascendente apresentava duas tênias: lateral e medial, em continuidade com as tênias homônimas do ceco, concordando com o descrito para o javali ${ }^{11}$ e para os suínos ${ }^{9,17}$.

O íleo do javali desembocava no intestino grosso exatamente na transição ceco-cólica, caracterizando uma junção íleoceco-cólica, concordando com o descrito para esta espécie ${ }^{11}$; para a espécie suína ${ }^{9,28,29,30}$ e para a espécie humana 5,7,31,32,33.

A forma papilar da proeminência ileal do javali correspondeu à descrita para esta espécie por Schäppi ${ }^{11}$; para o suíno 9,17,30,34,35 e à indicada, no indivíduo humano vivo $5,23,31,32,33,36,37,38,39,40,41,42,43,44$. Em nossa amostra identificamos, em $31,25 \%$ dos casos, uma "margem" proeminente da papila. Tal observação não correspondeu ao descrito para a espécie suína ${ }^{9,30}$, e poderíamos sugerir a existência de uma variação no javali, eventualmente um outro tipo de conformação da eminência ileal para esta espécie. Apesar de não termos observado a papila ileal no animal vivo, considerando o curto espaço de tempo entre a morte do animal e a coleta do material, permitimonos concordar com Prado 9 e Prado e Di Dio $^{30}$ e admitir que a forma papilar é a encontrada no animal vivo.

As dimensões da papila ileal dos javalis variaram, em seus contornos cranial e caudal, com valores de, respectivamente,
1,94 e 1,62 para as fêmeas e de 1,61 e 1,33 para os machos. Nossos dados estão próximos àqueles observados por Schäppi ${ }^{11}$, para esta espécie e para suínos ${ }^{9,30}$. O contorno cranial da papila ileal de javalis, mostrou-se significantemente maior do que o caudal. A diferença de altura entre os contornos da papila foi apontada, também, por outros autores. Ao tratar dos estágios evolutivos da válvula íleo-cecal em humanos, Struthers ${ }^{45}$ relacionou esta diferença com a angulação da terminação do íleo com o ceco. No javali, apesar de se formar um ângulo agudo entre estes dois órgãos, a papila, na maioria dos casos, dispunha-se para o lume cecal. Schäppi ${ }^{11}$ descreveu esta conformação em 100\% dos casos observados, entretanto seu trabalho inclui apenas cinco exemplares de javalis. Acreditamos que a variabilidade da disposição da papila encontrada em nossas observações deva-se a mobilidade da mesma diante a atividade intestinal, como descrito para suínos ${ }^{46} \mathrm{e}$, talvez, seja esta sua disposição em repouso, justificando, assim, a menor altura do contorno caudal.

A terminação do íleo no ceco, naqueles animais que possuem digestão parcial ou total neste órgão é muito importante, facilitando o esvaziamento do conteúdo ileal no ceco ${ }^{1}$. O javali, como o porco doméstico, apresenta digestão parcial no ceco e somos levados a concordar com as ponderações de Prado e Di Dio ${ }^{30}$, para suínos, que esta não seria prejudicada pela disposição ceco-cólica da terminação do íleo, devido a sua mobilidade ${ }^{46}$. 
Considerando que, na maioria dos casos, a papila ileal de javalis está direcionada para o lume cecal, podemos inferir que esta disposição contribui para o escoamento do conteúdo ileal no ceco.

Verificamos, no ápice da papila, um óstio central (ostium ileale), de forma estrelada ( $75 \%$ dos casos), com lobulações radiais, assemelhando-se com o descrito para o javali ${ }^{11}$ e para suínos ${ }^{9,30}$; ou de forma ovalada (25\% dos casos). Admitimos que a forma do óstio ileal do javali seja determinada pela projeção das pregas da mucosa da papila ileal, à semelhança do indicado para suínos ${ }^{9,30}$.

\section{Morphological characterization of the transition between the small and the large intestines in boar (Sus scrofa, L., 1758)}

Key words:

Morphology. Intestines. Wild boar. Anatomy. Boar. Sus scrofa (L., 1758).

morphology of the transition between small and large intestines was carried out in 32 wild boars (Sus scrofa, L., 1758) genetically chosen, male and female, with ages averaging from young ( 4 months) to adult (14 months). We examed at macroscopic level all the specimens - the shape and direction of the ileal prominence into the large intestine, of the ostium ileale, of the termination of the ileum and of the intestines ceco and ascending colon. Then, whe took some measurements of the ileum inside the large intestine. The results were analyzed by Wilcoxon Test (a $f, 0,05)$. The terminal ileum showed cylindrical morphology in $100 \%$ of the cases. In all of the cases, we observed that the ileal eminence was papilar shape - ileal papilla (papilla ilealis) towards the large intestine with an oblique direction. On the top of the papilla we observed one central ostium (ostium ileale), relatively closed and star shaped ( $75 \%$ of the cases), or relatively opened and oval shaped $(25 \%)$. Our findings demonstrate that the different morphology of the ileo-ceco-colic junction as compared to other animals, another indication against the generalization of the description of this region in mammals.

\section{Referências}

1 PELCKMANS, P. A. Study on the sphincteric nature of the ileocecal junction. A morphological and pharmacological approach. 1988. 182 p. Thesis
(Doctoral) - Universiteit Antwerpen, Universitaire Instelling Antwerpen, Departement Genneskunde, Antwerpen, 1988.

2 GROVUM, W. L.; WILLIAMS, V. H. Rate of passage of digesta in sheep. British Journal of Nutrition, v. 29, p. 13-21, 1973. 
3 JANKOWSKI, J. A.; GOODLAD, R. A. ; WRIGHT, $\mathrm{N}$. A. Maintenance of normal intestinal mucosa: function, structure and adaptation. Gut, v. 35, n. 1, p. S1-4, 1994. Supplement.

4 WENK, C. The role of dietary fibre in digestive physiology of the pig. Animal Feed Science and Technology, v. 90, p. 21-33, 2001.

5 Di Dio, L.J.A. Dados anatômicos sobre o "piloro" íleo-ceco-cólico (com observação in vivo da "papila" íleo-ceco-cólica). Revista do Hospital Nossa Senhora da Aparecida, São Paulo, v. 5, n. 4, p. 191-442, 1952.

6 KUMMAR, D.; PHILLIPS S. F. The contribution of external ligamentous attachments to function of the ileocecal junction. Diseases of the Colon and Rectum, v. 30, n. 6 , p. $410-416,1987$

7 SMITH-AGREDA, J. Arquitectura funcional de la región ileocaecal. Anales de Anatomia, v. 10, n. 21, p. 461-506, 1961

8 McCANCE, R. A. The effect of the age on the weights and lengths of pig's intestines. J. Anat., n. 117, p. 475479 .

9 PRADO, I. M. M. Macro- e mesoscopia da junção íleo-ceco-cólica do Sus scrofa domestica (Linnaeus, 1758). 1996. 173 f. Dissertação (Mestrado em Anatomia dos Animais Domésticos) - Faculdade de Medicina Veterinária e Zootecnia, Universidade de São Paulo, São Paulo, 1996.

10 PRADO, I. M. M.; MIGLINO, M. A.; DI DIO, L. J. A. Morphology of the swine ileum terminale. Annals of Anatomy, v. 179, p. 475-479, 1997.

11 SCHÄPPI, E. Magen und Darm des Wildeschweines. 1931. 38 p. Inaugural-Dissertation. VeterinarinärMedizinischen Fakultät der Universität Zürich, Zürich, 1931.

12 SIEGEL, S. Estadistica no parametrica. México: Trillas, 1975. 346 p.

13 INTERNATIONAL COMMITTEE ON VETERINARY GROSS ANATOMICAL NOMENCLATURE. Nomina anatomica veterinaria. 4. ed. Zurich, 1994. (Together with nomina histologica, 2.ed., 1992 and nomina embriologica veterinaria, 1992).

14 BOURDELLE, E. Dissection de la region abdominale. In: MONTANÉ, L.; BOURDELLE, E. Anatomie régionale des animaux domestiques. Paris: J. B. Baillière, 1920. v. III, p. 268-275.

15 GONZALEZ Y GARCIA, J.; GONZALEZ-ALVAREZ, R. Anatomia comparada de los animales domesticos. 7. ed. Madrid: Gráficas Canales, 1961. p. 472-497.

16 SISSON, S.; GROSSMAN, J. D. Anatomia de los animales domesticos. 4. ed. Barcelona: Salvat, 1972. $474 \mathrm{p}$.

17 SISSON, S. Sistema digestivo do suíno. In: GETTY, R. Sisson/Grossman anatomia dos animais domésticos.
5. ed. Rio de Janeiro: Interamericana, 1981. p. 11961199.

18 CUTORE, G. Sul comportamento della tonaca muscolare in corrispondenza della valvola del colon. Ricerche morfologiche. Archivio Italiano di Anatomia e di Embriologia, v. 30, n. 1, p. 72-93, 1932b.

19 MARTIN, E. The ileocolic valve as a factor in chronic intestinal stasis; operative treatment. Transactions of the American Surgical Association, v. 32, p. 175-186, 1914.

20 BUSI, A. La rappresentazione radiografica della valvola ileo-colica (Valvola del Varolio). Bolletino della Societa Medica, a.91, v. 9-8, n. 4-5, p. 210-214, 1920a.

21 . La rappresentazione radiografica della valvola ileo-colica (Valvola del Varolio). In: CONGR. ITAL. D. RADIOL. MED. MILANO, 3., 1920b. [s.I.] Atti...

22 BUSTOS, J. M. O. El organo antrobauhiniano (su fisiopatologia). In: JORNADA PAN-AMERICANA DE GASTRENTEROLOGIA, 2., 1951, Rio de Janeiro. Anais. Rio de Janeiro: [s.n.]. 1951. p. 420-430.

23 DI DIO, L. J. A.; CARRIL, C. F. Observações sobre o mecanismo do piloro íleo-ceco-cólico em indivíduo vivo com a papila íleo-ceco-cólica exteriorizada. Revista Brasileira de Gastroenterologia, v. 6, n. 1, p. 67-96, 1954.

24 MÜLLER, G.; SMITH-AGREDA, J. Form und funktion der regio ileocaecalis. Morphologisches Jahrbuch, v. 104, p. 1-26, 1963.

25 ROSANOV, B. N.; JURASSOV, I. B. Insuficiência da válvula de Bauhin. Vestnik Khirurgii, v. 11, n. 31, p. 3-16, 1927.

26 RUOTOLO, A. Le basi anatomiche dell'ampolla intestinale di Busi. Quaderni di Anatomia Pratica, v. 5 , n. $1-4$, p. 340-352, 1950.

27 WALCKER, F. I. Die Bedeutung der Bauhinischen und appendikulären Klappe. Experimentelle und anatomische Untersuchungen. Archiv fuer klinische Chirurgich, v. 170, p. 700-721, 1932.

28 PRADO, I. M. M.; DI DIO, L. J. A. Arrangement of the tunica muscularis in the ileal papilla of swine. Annals of Anatomy, v. 181, p. 339-344, 1999.

29 PRADO, I. M. M. Pesquisas microscópicas da junção íleo-ceco-cólica de suínos, em especial sobre os componentes muscular e colágeno e suas relações funcionais. 2001. $117 \mathrm{f}$. Tese (Doutorado em Anatomia dos Animais Domésticos) - Faculdade de Medicina Veterinária e Zootecnia, Universidade de São Paulo, São Paulo, 2001.

30 PRADO, I. M. M.; DI DIO, L. J. A. The "human"like morphology of the swine papilla ilealis. Annals of Anatomy, v. 182, p. 371-375, 2000.

31 DI DIO, L. J. A. Anatomia dos piloros (esfincteres) do sistema digestório. Parte geral. Revista de Medicina de São Paulo, v. 32, p. 273-280, 1948. 
32 _. Anatomia dos piloros (esfincteres) do sistema digestório. Parte especial. Revista de Medicina de São Paulo, v. 33, p. 43-61, 1949

33 . Piloros do sistema digestório. Revista do Hospital Nossa Senhora da Aparecida, São Paulo, v. 3, n. 2, p. 55-104, 1950.

34 DYCE, K. M.; SACK, W. O.; WENSING, C. J. D. Tratado de anatomia veterinária. Rio de Janeiro: Guanabara Koogan, 1987. 522 p.

35 NICKEL, R.; SCHUMMER, A.; SEIFERLE, E. The viscera of the domestic mammals. 2. ed. Berlim: Parey, 1979. p. 137-146.

36 ABREU, A.; DI DIO, L. J. A.; LIMA, W. L.; PINTO, E. P. Observação "in vivo" da papila ileocecocolica. Revista da Associação Médica Brasileira, v. 2, n. 1, p. 30-32, 1955.

37 BROUCÁ, R. Contribución al estudio de la válvula ileo-cecal. Boletines y Trabajos de la Sociedad de Cirurjanos, Buenos Ayres, v. 8, p. 439-443, 1924a.

38 . La valvula ileo-cecal. Su concepto esfinteriano. Revista de la Asociacion Medica Argentina, v. 37, p. $315-326,1924 b$.

39 La valvula ileo-cecal. Su concepto esfinteriano Semana Medica, v. 32, p. 800-822, 1925.

40 FLEISCHNER, F. G.; BERNSTEIN, C. Roentgenanatomical studies of the normal ileocecal valve. Radiology, v. 54, n. 1, p. 43-58, 1950.

41 GRASSO, R. L'endoscopia dello sfintere ileo-cecale e la sulla strutura nel vivente. Arch. Ital. Mal. App. Dig., v. 2, p. 400-414, 1933.

42 RUTHERFORD, A. H. The ileo-caecal valve. London: H.K. Lewis, 1914. 63 p.

43 . The frenula valvulae coli. Journal of Anatomy, v. 60, n. 4, p. 411-415, 1926.

44 ULIN, A. W.; DEUTSCH, J. Visualization of ileocecal papilla in a living subject. Gastroenterology, v. 16, n. 2, p. 444-449, 1950.

45 STRUTHERS, J. On varieties of the appendix vermiformis, caecum and ileo-colic valve in man. Edinb. Med. J., v. 39, p. 289-306, 438-451, 1893.

46 RAYNER, V.; WENHAM, G. Small intestinal motility and transit by electromyography and radiology in the fasted and fed pig. Journal of Physiology, v. 379, p. 245-256, 1986. 